\title{
Síntomas de ansiedad, depresión y conductas de autocuidado durante la pandemia de COVID-19 en la población general
}

\author{
Oscar Galindo-Vázquez, ${ }^{*}$ Mónica Ramírez-Orozco, ${ }^{2}$ Rosario Costas-Muñiz, ${ }^{3}$ \\ Luis A. Mendoza-Contreras, ${ }^{1}$ Germán Calderillo-Ruíz ${ }^{4}$ y Abelardo Meneses-García ${ }^{5}$ \\ ${ }^{1}$ Instituto Nacional de Cancerología, Servicio de Psicooncología, Ciudad de México, México; ${ }^{2}$ Universidad Nacional Autónoma de México, Facultad \\ de Psicología, Ciudad de México, México; ${ }^{3}$ Memorial Sloan-Kettering Cancer Center, Departamento de Psiquiatría y Ciencias del Comportamiento, \\ Nueva York, Estados Unidos; ${ }^{4}$ Instituto Nacional de Cancerología, Subdirección de Medicina Interna, Ciudad de México, México; ${ }^{5}$ nstituto Nacional \\ de Cancerología, Dirección General, Ciudad de México, México
}

\section{Resumen}

Introducción: La pandemia por COVID-19 puede tener consecuencias psicosociales importantes en la población. Objetivo: Determinar los niveles de síntomas de ansiedad, depresión y conductas de autocuidado durante la pandemia de COVID-19 en población general. Método: Encuesta en línea distribuida durante tres semanas mediante muestreo no probabilístico. Se empleó el Cuestionario sobre la Salud del Paciente PHQ-9, la Escala del Trastorno de Ansiedad Generalizada GAD-7 y la Escala análoga visual de conductas de autocuidado. Se realizaron análisis descriptivos y de comparación entre los grupos con ansiedad y depresión. Resultados: Se incluyeron 1508 participantes, 20.8 \% presentó síntomas de ansiedad grave y $27.5 \%$, síntomas de depresión grave. Ser mujer, soltero(a) no tener hijos, presentar comorbilidad médica y antecedentes de atención a la salud mental estuvieron relacionados con la presencia de mayores niveles de síntomas de ansiedad y depresión; 66 a 80 \% de la población cumplía con las recomendaciones de autocuidado. Se identificó la necesidad de recibir atención de salud mental. Conclusión: Se observó mayor número de individuos con síntomas de ansiedad y depresión moderadas a graves que en otras pandemias. Los efectos psicológicos de la pandemia de COVID-19 se consideran un problema de salud mental pública emergente, por lo que se recomienda la implementación de programas para su atención.

PALABRAS CLAVE: Ansiedad. COVID-19. Depresión. Pandemia.

\section{Symptoms of anxiety, depression and self-care behaviors during the COVID-19 pandemic in the general population}

\begin{abstract}
Introduction: The COVID-19 pandemic can have important psychosocial consequences in the population. Objective: To determine the levels of anxiety, depression and self-care symptoms during the COVID-19 pandemic in the general population. Method: Online survey distributed over three weeks using a non-probability sampling. The PHQ-9 Patient Health Questionnaire, the GAD-7 Generalized Anxiety Disorder Scale and Visual analog scale for self-care behaviors were used. Between-group (anxiety and depression) descriptive and comparison analyses were carried out. Results: Out of 1508 included participants, $20.8 \%$ had symptoms of severe anxiety, while $27.5 \%$ showed symptoms of severe depression. Being a woman, being single, having no children, having medical comorbidities and a history of mental health care were risk factors for developing symptoms of anxiety and depression; 66 to $80 \%$ of the population complied with self-care recommendations. A need for receiving mental health care was identified in our study population. Conclusion: $A$ larger number of individuals with moderate to severe
\end{abstract}

Correspondencia:

*Oscar Galindo-Vázquez

E-mail: psigalindo@yahoo.com.mx
Gac Med Mex. 2020;156:298-305

Disponible en PubMed

www.gacetamedicademexico.com

0016-3813/@ 2020 Academia Nacional de Medicina de México, A.C. Publicado por Permanyer. Este es un artículo open access bajo la licencia CC BY-NC-ND (http://creativecommons.org/licenses/by-nc-nd/4.0/). 
anxiety and depression symptoms were observed than in other pandemics. COVID-19 pandemic psychological effects are considered an emerging public health problem, and implementation of programs for their care is therefore recommended.

KEY WORDS: Anxiety. COVID-19. Depression. Pandemic.

\section{Introducción}

La pandemia por COVID-19 ha traído no solo el riesgo de contagios y muertes por infección, sino también efectos psicológicos importantes. ${ }^{1}$ Se conoce que los factores psicológicos desempeñan un papel vital en el éxito de las estrategias de salud pública utilizadas para controlar epidemias y pandemias; así como en la comunicación de riesgos, vacunación y terapia antiviral, prácticas de higiene y distanciamiento social..$^{2-4}$

Se ha reportado que las pandemias, como la del síndrome respiratorio agudo severo (SARS), son situaciones estresantes que amenazan la salud física y el bienestar psicológico, además de causar interrupciones en las funciones interpersonales y la percepción de que el contagio es relativamente incontrolable aun cuando se lleven a cabo medidas que reducen el riesgo (por ejemplo, usar mascarillas, evitar aglomeraciones). ${ }^{5}$

En los brotes virales, una persona con ansiedad grave puede malinterpretar dolores musculares benignos o tos como signos de infección, así como presentar comportamientos desadaptativos como lavarse las manos compulsivamente, retraimiento social y realizar compras de pánico, que pueden tener consecuencias negativas para el individuo y su comunidad. Por ejemplo, la sensación de urgencia por productos necesarios para la cuarentena puede llevar a gastar demasiado en el almacenamiento de recursos y perjudicar a la comunidad, la cual necesita dichos recursos para otros fines, incluida la atención médica. ${ }^{3}$ Por otra parte, las personas que se consideran con bajo riesgo de infección es poco probable que cambien su comportamiento y sigan las recomendaciones de distanciamiento social, con el consecuente impacto negativo en los esfuerzos por mitigar la propagación del virus.

En relación con los síntomas psicológicos reportados, en $21.3 \%$ de 7143 estudiantes universitarios expuestos a COVID-19 se identificó ansiedad leve; en $2.7 \%$, ansiedad moderada y en $0.9 \%$, ansiedad grave. Vivir en áreas urbanas y con los padres fueron factores protectores contra la ansiedad. Tener familiares 0 conocidos infectados de COVID-19, situación económica inestable y retrasos en las actividades académicas se asociaron a mayor ansiedad $(p<0.001)$. El apoyo social se correlacionó negativamente con el nivel de ansiedad $(p<0.001){ }^{1}$

Después de un mes de recuperación, en $35 \%$ de 180 sobrevivientes de SARS se identificaron síntomas de ansiedad y/o depresión moderados a graves 0 graves. Los trabajadores de la salud o quienes tenían familiares fallecidos por SARS fueron más propensos a desarrollar niveles altos de angustia. ${ }^{6}$

Al comparar a trabajadores de la salud hospitalarios $(n=82)$ durante el pico de la epidemia y personal del hospital que se recuperó de SARS $(n=97)$, se identificó que ambos grupos presentaron el mismo nivel de preocupación de infectar a otros (especialmente a los miembros de su familia). Los trabajadores tenían más miedo relacionado con la infección; en los sobrevivientes, el miedo relacionado con el SARS se correlacionó con síntomas de estrés postraumático; además, manifestaron preocupación por otros problemas de salud y la discriminación $(p<0.05) .^{7}$ Las personas que han experimentado emergencias de salud pública tienen diversos grados de estrés, incluso después de que el evento ha terminado o se han recuperado y egresado del hospital. ${ }^{6,8}$

Teniendo en cuenta los límites de interacción social y las medidas de confinamiento, los servicios de salud mental han adoptado el uso de líneas directas, plataformas de aplicaciones móviles, internet y redes sociales para compartir estrategias para lidiar con el estrés, ${ }^{9}$ así como para evaluar el impacto psicosocial en la población expuesta. Por lo tanto, el objetivo de esta investigación fue determinar los niveles de síntomas de ansiedad y de depresión, así como conductas de autocuidado durante la pandemia de COVID-19 en población general.

\section{Método}

Se realizó un estudio no experimental, transversal, ${ }^{10}$ para el cual se realizó una encuesta en línea mediante un muestreo no probabilístico por conveniencia; se incluyeron 1508 participantes, hombres y mujeres de México y el extranjero. Como criterios de inclusión se consideró edad mínima de 12 años y saber leer y escribir. Los individuos con deterioro cognitivo que les impidiera contestar la encuesta fueron excluidos y fueron eliminados de la investigación quienes durante 
o después del llenado de la encuesta decidieron no continuar participando.

Se diseñó una cédula de identificación que incluía datos sociodemográficos y clínicos. Se emplearon los siguientes instrumentos de evaluación:

- Cuestionario sobre la Salud del Paciente (PHQ-9). EI PHQ-9 (Patient Health Questionnaire-9), desarrollado por Kroenke et al. en 2001,11 es una herramienta de tamizaje que evalúa la posible presencia de trastorno depresivo mayor y la gravedad de los síntomas de depresión. Su estructura es unidimensional, cuenta con nueve reactivos basados en los criterios del DSM IV TR (Statistical Manual of Mental Disorders, Fourth edition, Text Revision) y un alfa de Cronbach global de 0.89 . Fue validado en la población mexicana, ${ }^{*}$ con una consistencia interna de 0.86 y una varianza explicada de $47 \%$.

- Escala del Trastorno de Ansiedad Generalizada (GAD-7, Generalized Anxiety Disorder-7). Desarrollada por Spitzer et al. en 2006, ${ }^{12}$ se trata de una herramienta de tamizaje que evalúa la presencia de posible trastorno de ansiedad generalizada. Cuenta con una estructura unidimensional de siete reactivos basados en los criterios del DSM IV TR, que explican $63 \%$ de la varianza y un alfa de Cronbach global de 0.92 . Fue validada en población mexicana, ${ }^{*}$ con una consistencia interna de 0.88 y una varianza explicada de $57.72 \%$.

- Escala visual análoga de conductas de autocuidado. Las conductas se valoraron mediante una escala visual análoga de 10 puntos, en la que 0 significa "para nada sigo la recomendación" y 10 , "sigo la recomendación todo el tiempo", que especifican cómo los individuos realizaban las estrategias de autocuidado.

La recolección de la muestra se llevó a cabo del 26 de marzo al 12 de abril de 2020. Por medio electrónico se explicó a cada participante el objetivo de la investigación, procedimiento, confidencialidad de los datos, así como los posibles riesgos y beneficios. Todos los individuos participaron voluntariamente y concedieron su consentimiento por escrito. Los procedimientos de esta investigación cumplieron con las

* Mendoza-Contreras L, Flores-Juárez J, Núñez-Hernández J, Guzmán Saldaña R, Lerma A. Galindo-Vázquez O. Propiedades psicométricas del Cuestionario sobre la Salud del Paciente (PHQ-9) y de la Escala del Trastorno de Ansiedad Generalizada (GAD-7) en población general mexicana. México: Servicio de Psicooncología, Instituto Nacional de Cancerología; 2020. Documento no publicado. disposiciones de la Declaración de Helsinki respecto a la investigación en humanos.

Los datos se analizaron con SPSS versión 22.0. Se realizó análisis descriptivo de medidas de tendencia central y dispersión para ilustrar las características demográficas y clínicas, así como análisis univariado para identificar las diferencias entre las variables sociodemográficas y el nivel de los síntomas de ansiedad y depresión. La normalidad de las variables se determinó por medio de la prueba de bondad de ajuste de Kolmogorov-Smirnov ( $p<0.001$ ), con la que se observó una distribución no normal, por lo que se utilizaron medianas y las pruebas no paramétricas $U$ de Mann-Whitney y de Kruskal-Wallis. ${ }^{13}$ Finalmente, se realizó análisis de correlación de Pearson. Se estableció un valor de $p<0.05$ como significativo estadísticamente.

\section{Resultados}

Como se observa en la Tabla 1, la muestra total estuvo integrada por 1508 participantes, 1123 mujeres y 385 hombres, con edad promedio de 34 años; $61.3 \%$ sin hijos, $50.8 \%$ soltero, $55.2 \%$ con licenciatura, $35.6 \%$ se desempeñaba laboralmente como profesional y $24 \%$ mencionó tener alguna enfermedad crónico-degenerativa.

Se exploraron diferentes áreas comportamentales relacionadas con la contingencia y sus consecuencias psicosociales. La mayoría de los participantes (92\%) refirió que sí se realizaría la prueba para la detección de COVID-19, en tanto que $90 \%$ no contaba en ese momento con algún familiar o amistad con infección por el virus.

Respecto a las conductas de autocuidado, destacó una adecuada adhesión a las recomendaciones, ya que $80 \%$ cumplía con no asistir a reuniones o sitios conglomerados, $88 \%$ lavaba o desinfectaba sus manos de forma frecuente, $66 \%$ mantenía la distancia recomendada (1.5 a 2 m) y $72 \%$ se quedaba en casa.

En relación con las estrategias de afrontamiento, $41 \%$ se preocupaba poco por enfermar, aproximadamente $15 \%$ se preocupaba de forma frecuente por contagiarse, mientras que $31 \%$ analizaba continuamente sus sensaciones corporales y las interpretaba como síntomas de la enfermedad. La mitad de los participantes usó frecuentemente estrategias de experiencias estresantes pasadas para reducir el miedo y generó una lista de actividades para mantenerse con actividad; el mismo porcentaje aseguró mantener una actitud optimista y objetiva ante la 
Tabla 1. Características de los individuos encuestados en relación a síntomas depresión y ansiedad ante la pandemia de COVID-19 ( $n=1508)$

\begin{tabular}{|c|c|c|c|c|c|}
\hline \multicolumn{3}{|l|}{ Edad (años) } & \multicolumn{3}{|l|}{$\overline{\mathrm{X}}=34.46$, rango $18-82$} \\
\hline & $n$ & $\%$ & & $\mathrm{n}$ & $\%$ \\
\hline $\begin{array}{l}\text { Sexo } \\
\text { Mujer } \\
\text { Hombre }\end{array}$ & $\begin{array}{c}1123 \\
385\end{array}$ & $\begin{array}{r}74.5 \\
25.5\end{array}$ & $\begin{array}{l}\text { Escolaridad } \\
\text { Educación básica } \\
\text { Bachillerato }\end{array}$ & $\begin{array}{l}34 \\
209\end{array}$ & $\begin{array}{l}2.3 \\
13.9\end{array}$ \\
\hline $\begin{array}{l}\text { País } \\
\text { México } \\
\text { Otro }\end{array}$ & $\begin{array}{c}1421 \\
87\end{array}$ & $\begin{array}{c}94.2 \\
5.8\end{array}$ & $\begin{array}{l}\text { Licenclatura } \\
\text { Posgrado } \\
\text { Otro }\end{array}$ & $\begin{array}{c}833 \\
406 \\
26\end{array}$ & $\begin{array}{c}55.2 \\
26.9 \\
1.7\end{array}$ \\
\hline $\begin{array}{l}\text { Estado civil } \\
\text { Soltero } \\
\text { Casado } \\
\text { Viudo } \\
\text { Divorciado o separado } \\
\text { Unión libre } \\
\text { Otro }\end{array}$ & $\begin{array}{c}817 \\
397 \\
17 \\
111 \\
157 \\
9\end{array}$ & $\begin{array}{c}54.2 \\
26.3 \\
1.1 \\
7.4 \\
10.4 \\
0.6\end{array}$ & $\begin{array}{l}\text { Ocupación } \\
\text { Hogar } \\
\text { Estudiante } \\
\text { Empleado } \\
\text { Desempleado } \\
\text { Profesional } \\
\text { Jubilado }\end{array}$ & $\begin{array}{c}58 \\
256 \\
254 \\
48 \\
356 \\
27\end{array}$ & $\begin{array}{c}5.8 \\
25.6 \\
25.4 \\
4.8 \\
35.6 \\
2.7\end{array}$ \\
\hline $\begin{array}{l}\text { Paternidad } \\
\text { Sí } \\
\text { No }\end{array}$ & $\begin{array}{l}583 \\
925\end{array}$ & $\begin{array}{l}38.7 \\
61.3\end{array}$ & $\begin{array}{l}\text { Residencia en México }(n=1426) \\
\text { Ciudad de México } \\
\text { Estado de México } \\
\text { Otros estados }\end{array}$ & $\begin{array}{l}688 \\
265 \\
473\end{array}$ & $\begin{array}{l}42.8 \\
18.6 \\
33.2\end{array}$ \\
\hline \multirow[t]{2}{*}{$\begin{array}{l}\text { Comorbilidad médica }(n=417) \\
\text { Hipertensión } \\
\text { Diabetes } \\
\text { Cáncer } \\
\text { Depresión } \\
\text { Ansiedad } \\
\text { Otra }\end{array}$} & $\begin{array}{c}85 \\
43 \\
29 \\
75 \\
116 \\
196\end{array}$ & $\begin{array}{c}20.4 \\
10.3 \\
7.0 \\
18 \\
27.8 \\
47.0\end{array}$ & $\begin{array}{l}\text { Enfermedad } \\
\text { Sí } \\
\text { No } \\
\text { Seguro médico } \\
\text { Sí } \\
\text { No }\end{array}$ & $\begin{array}{c}400 \\
1108\end{array}$ & $\begin{array}{l}26.5 \\
73.5\end{array}$ \\
\hline & & & $\begin{array}{l}\text { Atención de salud mental previa } \\
\text { Sí } \\
\text { No }\end{array}$ & $\begin{array}{l}929 \\
579\end{array}$ & $\begin{array}{l}61.6 \\
38.4\end{array}$ \\
\hline
\end{tabular}

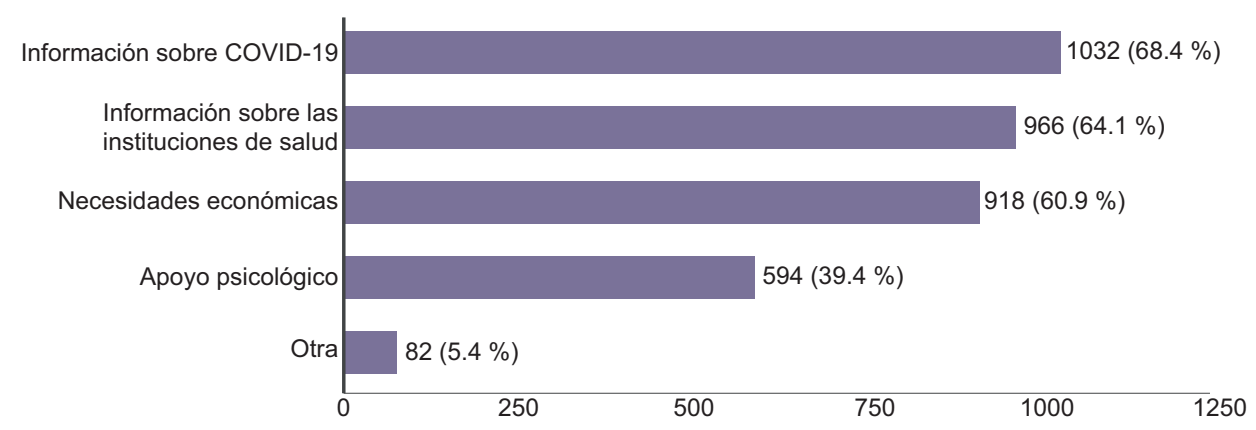

Figura 1. Aspectos percibidos para adaptarse a la pandemia de COVID-19.

situación, así como contar con redes de apoyo para hablar y solucionar problemas (Tabla 2).

En cuanto a las necesidades específicas para enfrentar la problemática de salud actual, $68 \%$ respondió que tener información sobre la enfermedad era indispensable, conocer las instituciones de salud a las que puede acudir y cubrir las necesidades económicas de subsistencia; 34 \% consideró necesario atender el aspecto psicológico (Figura 1).

Las puntuaciones de los síntomas de ansiedad y depresión tuvieron medias de 12.35 y 14.4, respectivamente. Resaltó que $20.8 \%$ presentaba síntomas de ansiedad grave y $27.5 \%$, de depresión grave (Tabla 3). Se observó que los participantes sin hijos, con 
Tabla 2. Afrontamiento y conductas de autocuidado ante la pandemia de COVID-19 en individuos encuestados en relación con síntomas depresión y ansiedad

\begin{tabular}{|c|c|c|c|c|c|c|c|c|c|c|}
\hline & \multicolumn{2}{|c|}{ Nunca } & \multicolumn{2}{|c|}{$\begin{array}{l}\text { Pocas } \\
\text { veces }\end{array}$} & \multicolumn{2}{|c|}{ Frecuentemente } & \multicolumn{2}{|c|}{$\begin{array}{l}\text { Casi todo } \\
\text { el tiempo }\end{array}$} & \multicolumn{2}{|c|}{$\begin{array}{l}\text { Todo el } \\
\text { tiempo }\end{array}$} \\
\hline & $n$ & $\%$ & $n$ & $\%$ & $\mathrm{n}$ & $\%$ & $n$ & $\%$ & $\mathrm{n}$ & $\%$ \\
\hline $\begin{array}{l}\text { ¿Con qué frecuencia se preocupa por contagiarse de } \\
\text { COVID-19? }\end{array}$ & 7.4 & 6.9 & 629 & 41.7 & 523 & 34.7 & 164 & 10.9 & 81 & 5.4 \\
\hline $\begin{array}{l}\text { ¿Se encuentra continuamente analizando e interpretando sus } \\
\text { sensaciones corporales como síntomas de enfermedad? }\end{array}$ & 329 & 21.8 & 704 & 46.7 & 340 & 22.5 & 84 & 5.6 & 51 & 3.4 \\
\hline $\begin{array}{l}\text { ¿Se siente frustrado por los efectos que ha tenido COVID-19 en } \\
\text { su vida? }\end{array}$ & 207 & 13.7 & 585 & 38.8 & 436 & 28.9 & 185 & 12.3 & 95 & 6.3 \\
\hline $\begin{array}{l}\text { Cuando siente miedo, se apoya en las experiencias que ha } \\
\text { tenido en situaciones similares para reducir el miedo }\end{array}$ & 140 & 9.3 & 361 & 23.9 & 437 & 29.0 & 365 & 24.2 & 205 & 13.6 \\
\hline $\begin{array}{l}\text { Genera una lista de actividades diarias y trata de mantenerme } \\
\text { ocupado (a) }\end{array}$ & 138 & 9.2 & 302 & 20.0 & 390 & 25.9 & 390 & 25.9 & 288 & 19.1 \\
\hline Mantiene una actitud optimista y objetiva ante la situación & 20 & 1.3 & 134 & 8.9 & 423 & 28.1 & 521 & 34.5 & 410 & 27.2 \\
\hline \multirow{4}{*}{$\begin{array}{l}\text { Cuenta con alguien en quien puede apoyarse o con quien } \\
\text { puede hablar de sus problemas }\end{array}$} & 40 & 2.7 & 200 & 13.3 & 244 & 16.2 & 341 & 22.6 & 683 & 45.3 \\
\hline & \multicolumn{10}{|c|}{ Puntuación en la escala visual análoga } \\
\hline & \multicolumn{2}{|c|}{$0-1$} & \multicolumn{2}{|c|}{ 2-3 } & \multicolumn{2}{|c|}{ 4-5 } & \multicolumn{2}{|c|}{ 6-7 } & \multicolumn{2}{|c|}{$8-10$} \\
\hline & n & $\%$ & $n$ & $\%$ & n & $\%$ & n & $\%$ & n & $\%$ \\
\hline $\begin{array}{l}\text { ¿Qué tanto ha seguido las siguientes recomendaciones? } \\
\text { - No asistir a reuniones sociales o sitios conglomerados } \\
\text { - Lavarse o desinfectarse las manos con frecuencia } \\
\text { - Mantenerse al menos } 1.5 \text { m de distancia de otras personas } \\
\text { - Quedarse en casa }\end{array}$ & $\begin{array}{l}44 \\
10 \\
64 \\
97\end{array}$ & $\begin{array}{l}2.9 \\
0.7 \\
4.3 \\
6.5\end{array}$ & $\begin{array}{l}44 \\
12 \\
46 \\
49\end{array}$ & $\begin{array}{l}2.9 \\
0.8 \\
3.3 \\
3.3\end{array}$ & $\begin{array}{c}82 \\
40 \\
167 \\
120\end{array}$ & $\begin{array}{c}5.4 \\
2.7 \\
11.1 \\
7.9\end{array}$ & $\begin{array}{c}92 \\
97 \\
225 \\
123\end{array}$ & $\begin{array}{c}6.8 \\
6.4 \\
26.3 \\
8.8\end{array}$ & $\begin{array}{l}1236 \\
1349 \\
1006 \\
1109\end{array}$ & $\begin{array}{l}82.0 \\
89.4 \\
66.8 \\
73.0\end{array}$ \\
\hline
\end{tabular}

Tabla 3. Nivel de ansiedad y depresión derivadas de la pandemia de COVID-19

\begin{tabular}{|l|c|c|l|c|c|}
\hline \multicolumn{3}{|c|}{ Ansiedad } & \multicolumn{3}{c|}{ Depresión } \\
\hline Nivel & n & $\%$ & Nivel & n & $\%$ \\
\hline Mínima & 525 & 34.8 & Mínima & 598 & 39.7 \\
\hline Leve & 253 & 16.8 & Leve & 337 & 22.3 \\
\hline Moderada & 416 & 27.6 & Moderada & 158 & 10.5 \\
\hline Grave & 314 & 20.8 & Grave & 415 & 27.5 \\
\hline Total & 1508 & 100.0 & Total & 1508 & 100.0 \\
\hline
\end{tabular}

enfermedad médica y antecedentes de atención a la salud mental presentaban mayores niveles de depresión y ansiedad $(p<0.001)$. Específicamente el sexo femenino reportó mayores niveles de ansiedad y los individuos solteros, mayores niveles de depresión $(p<0.001)$ (Tablas 4 y 5$)$.
Cabe destacar que puede existir variabilidad en los datos de cada país, pues la encuesta se efectuó en diferentes momentos de acuerdo con la fase epidemiológica de cada nación. A pesar de lo cual, $88.4 \%$ de los participantes consideró que experimentará repercusiones negativas en su economía individual.

Se identificó una correlación positiva, de magnitud media y estadísticamente significativa $(r$ de Pearson $=0.721, p<0.001$ ), entre los niveles de los síntomas de depresión y ansiedad.

\section{Discusión}

Las principales estrategias para combatir el contagio por coronavirus COVID-19 implican conductas de autocuidado, las cuales deberían abordarse desde la perspectiva psicológica ya que requieren la modificación o implementación de conductas en las personas que aparentemente no tienen un reforzador inmediato, lo cual complica su ejecución. 
Tabla 4. Comparación de variables sociodemográficas de los participantes con síntomas de ansiedad $(n=1508)$

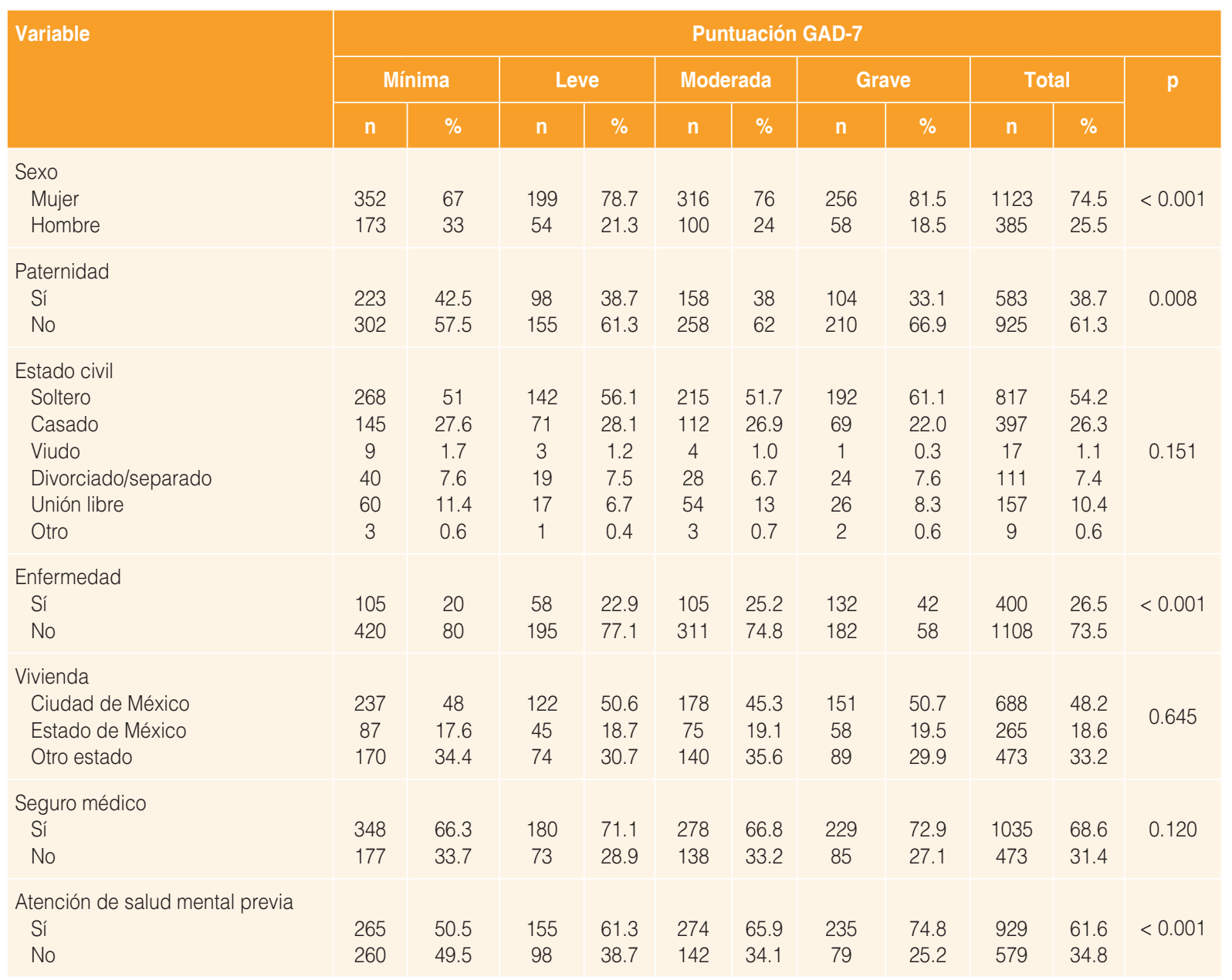

GAD-7 = Generalized Anxiety Disorder-7.

Se identificaron mayores niveles de ansiedad y depresión que los reportados en las pandemias de SARS e influenza, lo que denota mayor efecto en la salud mental de la población general. El sexo femenino, no tener hijos, estado civil soltero, comorbilidad médica y antecedentes de atención a la salud mental coincidieron con las variables indicadas en literatura como relacionadas con la presencia de mayores síntomas psicológicos; adicionalmente, se identificaron las preocupaciones económicas, las repercusiones de la pandemia en la vida diaria y el retraso académico. ${ }^{1}$ El $26.5 \%$ de la muestra reportó una enfermedad médica, principalmente de índole cardiometabólica, lo que significa que este grupo tiene mayor riesgo de enfermar gravemente de COVID-19 ${ }^{14} \mathrm{y}$ durante la pandemia pudiera enfrentarse a dificultades para obtener el tratamiento adecuado. ${ }^{9}$

Una posible explicación de los niveles altos de ansiedad y depresión es la alta exposición a información acerca de COVID-19, que Avittey relaciona con la exposición constante a titulares de noticias abrumadoras e información errónea. ${ }^{15}$

Se identificó la necesidad de información general acerca de las instituciones de salud a las que se puede acudir, así como la preocupación por los efectos de la pandemia de COVID-19 en la economía. La inestabilidad o disminución del ingreso familiar ha sido identificada como un factor significativo en la ansiedad durante la crisis. ${ }^{16}$

Aun cuando en el presente estudio se registró adherencia aceptable a las recomendaciones sanitarias, $5.8 \%$ no evitaba reuniones, $7.4 \%$ no guardaba la distancia adecuada entre personas y $9.8 \%$ continuaba saliendo de casa, situación que conlleva repercusiones en la salud pública, pues la diseminación y el contagio del virus se incrementan en la medida en que no se cumplen las estrategias de confinamiento y distanciamiento social. 
Tabla 5. Comparación de variables sociodemográficas entre participantes con síntomas de depresión $(\mathrm{n}=1508)$

\begin{tabular}{|c|c|c|c|c|c|c|c|c|c|c|c|}
\hline \multirow[t]{3}{*}{ Variable } & \multicolumn{11}{|c|}{ Puntuación PHQ-9 } \\
\hline & \multicolumn{2}{|c|}{ Mínima } & \multicolumn{2}{|c|}{ Leve } & \multicolumn{2}{|c|}{ Moderada } & \multicolumn{2}{|c|}{ Grave } & \multicolumn{2}{|c|}{ Total } & \multirow[t]{2}{*}{ p } \\
\hline & $\mathrm{n}$ & $\%$ & $\mathrm{n}$ & $\%$ & $\mathrm{n}$ & $\%$ & $\mathrm{n}$ & $\%$ & $\mathrm{n}$ & $\%$ & \\
\hline \multicolumn{12}{|l|}{ Sexo } \\
\hline Mujer & 429 & 71.7 & 247 & 73.3 & 127 & 80.4 & 320 & 77.1 & 1123 & 74.5 & 0.024 \\
\hline Hombre & 169 & 28.3 & 90 & 26.7 & 31 & 19.6 & 95 & 22.9 & 385 & 25.5 & \\
\hline \multicolumn{12}{|l|}{ Paternidad } \\
\hline Sí & 285 & 47.7 & 124 & 36.8 & 51 & 32.3 & 123 & 29.6 & 583 & 38.7 & $<0.001$ \\
\hline No & 313 & 52.3 & 213 & 63.2 & 107 & 67.7 & 292 & 70.4 & 925 & 61.3 & \\
\hline \multicolumn{12}{|l|}{ Estado civil } \\
\hline Soltero & 274 & 45.8 & 175 & 51.9 & 95 & 60.1 & 273 & 65.8 & 817 & 54.2 & \\
\hline Casado & 196 & 32.8 & 94 & 27.9 & 39 & 24.7 & 68 & 16.4 & 397 & 26.3 & \\
\hline Viudo & 10 & 1.7 & 1 & 0.3 & 0 & 0.0 & 6 & 1.4 & 17 & 1.1 & $<0.001$ \\
\hline Divorciado/separado & 45 & 7.5 & 23 & 6.8 & 9 & 5.7 & 34 & 8.2 & 111 & 7.4 & \\
\hline Unión libre & 69 & 11.5 & 42 & 12.5 & 14 & 8.9 & 32 & 7.7 & 157 & 10.4 & \\
\hline Otro & 4 & 0.7 & 2 & 0.6 & 1 & 0.6 & 2 & 0.5 & 9 & 0.6 & \\
\hline \multicolumn{12}{|l|}{ Enfermedad } \\
\hline Sí & 117 & 19.6 & 80 & 23.7 & 46 & 29.1 & 157 & 37.8 & 400 & 26.5 & $<0.001$ \\
\hline No & 481 & 80.4 & 257 & 76.3 & 112 & 70.9 & 258 & 62.2 & 1108 & 73.5 & \\
\hline \multicolumn{12}{|l|}{ Vivienda } \\
\hline Ciudad de México & 278 & 48.7 & 149 & 47.2 & 67 & 46.5 & 194 & 49.1 & 688 & 48.2 & \\
\hline Estado de México & 95 & 16.6 & 54 & 17.1 & 39 & 27.1 & 77 & 19.5 & 265 & 18.6 & 0.173 \\
\hline Otro estado & 198 & 34.7 & 113 & 35.8 & 38 & 26.4 & 124 & 31.4 & 473 & 33.2 & \\
\hline \multicolumn{12}{|l|}{ Seguro médico } \\
\hline Sí & 421 & 70.4 & 232 & 68.8 & 113 & 71.5 & 269 & 64.8 & 1035 & 68.6 & 0.100 \\
\hline No & 177 & 29.6 & 105 & 31.2 & 45 & 28.5 & 146 & 35.2 & 473 & 31.4 & \\
\hline \multicolumn{12}{|c|}{ Atención de salud mental previa } \\
\hline Sí & 296 & 49.5 & 224 & 66.5 & 106 & 67.1 & 303 & 73 & 929 & 61.6 & $<0.001$ \\
\hline No & 302 & 50.5 & 113 & 33.5 & 52 & 32.9 & 112 & 27 & 579 & 38.4 & \\
\hline
\end{tabular}

PHQ-9 = Patient Health Questionnaire-9.

Finalmente, si bien se identificaron adecuadas estrategias psicológicas para afrontar la pandemia de COVID-19, la mitad de los participantes no contaba con dichas herramientas ni condiciones para adaptarse a la situación, por lo que es necesario enfocarse en las necesidades particulares de la población y cubrirlas para ayudar a mejorar las estrategias de afrontamiento. El $24 \%$ de los participantes consideró necesario recibir atención de salud mental, sin embargo, $72 \%$ no disponía de algún servicio de atención a distancia, ya fuera telefónicamente o en línea.

Es relevante considerar recomendaciones como las reportadas por $\mathrm{Li}^{9}$ respecto a que la población expuesta a COVID-19 se puede clasificar en cuatro niveles:

1. Personas más vulnerables a problemas de salud mental, como pacientes hospitalizados con infección confirmada o condición física grave, profesionales de salud de primera línea y personal administrativo.
2. Pacientes aislados y en clínicas con síntomas de infección atípica.

3. Individuos con contactos nivel 1 y 2 , es decir, miembros de la familia, colegas, amigos y rescatistas.

4. Personas afectadas por las medidas de prevención y control de epidemias, las personas susceptibles y población general.

Entre las limitaciones debe destacarse que la muestra se recolectó por conveniencia y que se utilizó un diseño de investigación transversal, por lo que se sugiere hacer un seguimiento prospectivo, lo cual permitirá observar cambios en los síntomas y las medidas de seguridad conforme la situación de salud pública se modifica.

\section{Conclusiones}

Los problemas de salud mental en la población general durante la pandemia de COVID-19 representan 
un reto para el sistema de salud pública, por lo que se requieren intervenciones psicosociales válidas y confiables para identificar oportunamente la aparición e intensidad de los síntomas de depresión y ansiedad, así como para evaluar los efectos de las intervenciones psicosociales clínicas y comunitarias.

\section{Conflicto de intereses}

Los autores declaran no tener conflicto de intereses alguno.

\section{Financiamiento}

Los autores no recibieron patrocinio para llevar a cabo este artículo.

\section{Responsabilidades éticas}

Protección de personas y animales. Los autores declaran que para esta investigación no se realizaron experimentos en seres humanos ni en animales.

Confidencialidad de los datos. Los autores declaran que han seguido los protocolos de su centro de trabajo en cuanto a la publicación de datos de pacientes.

Derecho a la privacidad y consentimiento informado. Los autores obtuvieron el consentimiento informado de los participantes referidos en el artículo. Este documento obra en poder del autor de correspondencia.

\section{Bibliografía}

1. Cao W, Fang Z, Hou G, Han M, Xu X, Dong J, et al. The psychological impact of the COVID-19 epidemic on college students in China. Psychiatry Res. 2020;287:112934.

2. Taylor S, Asmundson GJ. Treating health anxiety: a cognitive-behavioral approach. EE. UU.: Guilford; 2004.

3. Asmundson GJ, Taylor S. How health anxiety influences responses to viral outbreaks like COVID-19: what all decision-makers, health authorities, and health care professionals need to know. J Anxiety Disord. 2020;71:102211.

4. Wheaton MG, Abramowitz JS, Berman NC, Fabricant LE, Olatunji BO. Psychological predictors of anxiety in response to the H1N1 (swine flu) pandemic. Cognit Ther Res. 2012;36:210-218.

5. Gan Y, Liu Y, Zhang Y. Flexible coping responses to severe acute respiratory syndrome-related and daily life stressful events. Asian J Soc Psychol. 2004;7:55-66.

6. Cheng SK, Wong CW, Tsang J, Wong KC. Psychological distress and negative appraisals in survivors of severe acute respiratory syndrome (SARS). Psychol Med. 2004;34:1187-1195.

7. Ho SM, Kwong-Lo RS, Mak CW, Wong JS. Fear of severe acute respiratory syndrome (SARS) among health care workers. J Consulting Clin Psychol. 2005; 73:344-349.

8. Fan $\mathrm{F}$, Long $\mathrm{K}$, Zhou $\mathrm{Y}$, Zheng $\mathrm{Y}$, Liu $X$. Longitudinal trajectories of post-traumatic stress disorder symptoms among adolescents after the Wenchuan earthquake in China. Psychol Med. 2015;45:2885-2896.

9. Li W, Yang Y, Liu ZH, Zhao YJ, Zhang Q, Zhang L, et al. Progression of mental health services during the COVID-19 outbreak in China. Int J Biol Sci. 2020;16:1732-1738.

10. Babbie E. Fundamentos de la investigación social. México: International Thomson; 2000.

11. Kroenke K, Spitzer RL Williams JBW. The PHQ-9: validity of a brief depression severity measure. J Gen Intern Med. 2001;16:606-613.

12. Spitzer RL, Kroenke K, Williams JBW, Löwe B. A brief measure for assessing generalized anxiety disorder: The GAD-7. Arch Intern Med. 2006;166:1092-1097.

13. Juárez F, Villatoro J, López E. Apuntes de estadística inferencial. México: Instituto Nacional de Psiquiatría Ramón de la Fuente; 2002.

14. Centers for Disease Control and Prevention [Internet]. EE. UU.: Enfermedad del coronavirus 2019; 2020.

15. Ayittey FK, Ayittey MK, Chiwero NB, Kamasah JS, Dzuvor C. Economic impacts of Wuhan 2019-nCoV on China and the world. J Med Virol. 2020;92.

16. Liu ZF. A study on the relationship between adverse family experiences in childhood and emotional and anxiety disorders. Dalian Medical University; 2013. 